\title{
Marrow failure with sideroblasts in an autoimmune haemolytic crisis
}

\author{
M. C. Allison \\ M.B., M.R.C.P.
}

M. T. JEHA
M.D.

Royal United Hospital, Combe Park, Bath BA1 3NG

\begin{abstract}
Summary
We report a case of autoimmune haemolytic anaemia complicated by ineffective erythropoiesis associated with florid sideroblastic change in the bone marrow.

KEY WORDS: pyridoxine, Evan's syndrome, autoimmune haemolytic anaemia, sideroblastic anaemia.

\section{Introduction}

A poor reticulocyte response to haemolysis can be a manifestation of secondary marrow aplasia, but a hypercellular erythroid marrow response with reticulocytopenia is also recognised. We report a case where autoimmune thrombocytopenia occurred 2 years before a haemolytic crisis. The latter was complicated by reticulocytopenia and an abundance of ring sideroblasts in the bone marrow. The changes reversed with pyridoxine and prednisolone therapy.
\end{abstract}

\section{Case report}

A 24-year-old man presented in February 1980 with autoimmune thrombocytopenic purpura. The marrow showed abundant megakaryocytes but was otherwise normal. Complement fixing antibody to platelets was demonstrated. He made a prompt and complete recovery with corticosteroids.

He presented again in September 1982 with a 3day history of jaundice and faintness. He had been on no medication. He was markedly pale and jaundiced but no splenomegaly or other abnormality was found. His haemoglobin was $3.8 \mathrm{~g} / \mathrm{dl}$ with $78 \times 10^{9} / 1(7 \%)$ reticulocytes. His white count and platelets were normal and autoimmune profile negative. The serum bilirubin was very raised (165 nmol/l, all unconjugated) and the Coombs' test strongly positive. Haptoglobins were absent and red cell folate was normal. He was cautiously transfused and commenced on prednisolone, $60 \mathrm{mg}$, and folic acid, $15 \mathrm{mg}$ daily. After 3 days and four units of packed red cells his haemoglobin was only $3.6 \mathrm{~g} / \mathrm{dl}$ and the reticulocyte count very low at $5 \times 10^{9} / 1(1 \%)$. Bone marrow aspirate was cellular with active erythropoiesis, increased iron stores and plentifulo ring sideroblasts. He was given a total of 10 further 3 units of packed red cells, prednisolone was increased to $100 \mathrm{mg}$ daily and pyridoxine prescribed. He then developed a prompt reticulocyte response and made a full recovery (Fig. 1). He was well 10 weeks lateric with a haemoglobin of $12.2 \mathrm{~g} / \mathrm{dl}$, and a normal serum bilirubin. Sideroblasts were no longer present in theo bone marrow.

\section{Discussion}

This patient displays Evan's syndrome, in whiôh $\overrightarrow{0}$ autoimmune haemolysis and thrombocytopenia 8 cur either simultaneously or in sequence. A popir reticulocyte response to haemolysis is usually due.to marrow aplasia or folic acid deficiency. In addition Conley et al. (1982) described five cases wherea reticulocytopenia occurred in association with an $\bar{Q}$ erythroid marrow. One of their patients was investigated with ferrokinetic studies and the characteristicso of the autoantibody determined. They concluded that the antibody prevented the release of reticulocytes into the blood.

We do not consider that the marrow changes in our patient represent recovery from an aplastic arrest before presentation. While we cannot exclude this possibility, it is unlikely because the reticulocyte count was lowest on the day when the marrow? aspirate showed erythroid hyperplasia and remainedo inappropriately low for the degree of haemolysis for several days subsequently.

We have found two reports on sideroblastosis in autoimmune haemolysis. McFadzean and Daviso (1947) described seven cases where iron staining granules and occasional ring forms were seen in then marrow; their presence correlated with the degree of haemolysis and yet the reticulocyte response remained good. Folic acid assays were not done. Celada, Farquet and Muller (1977) reported chronices haemolysis, reticulocytopenia and a sideroblastic marrow in an elderly patient on immunosuppressives. 


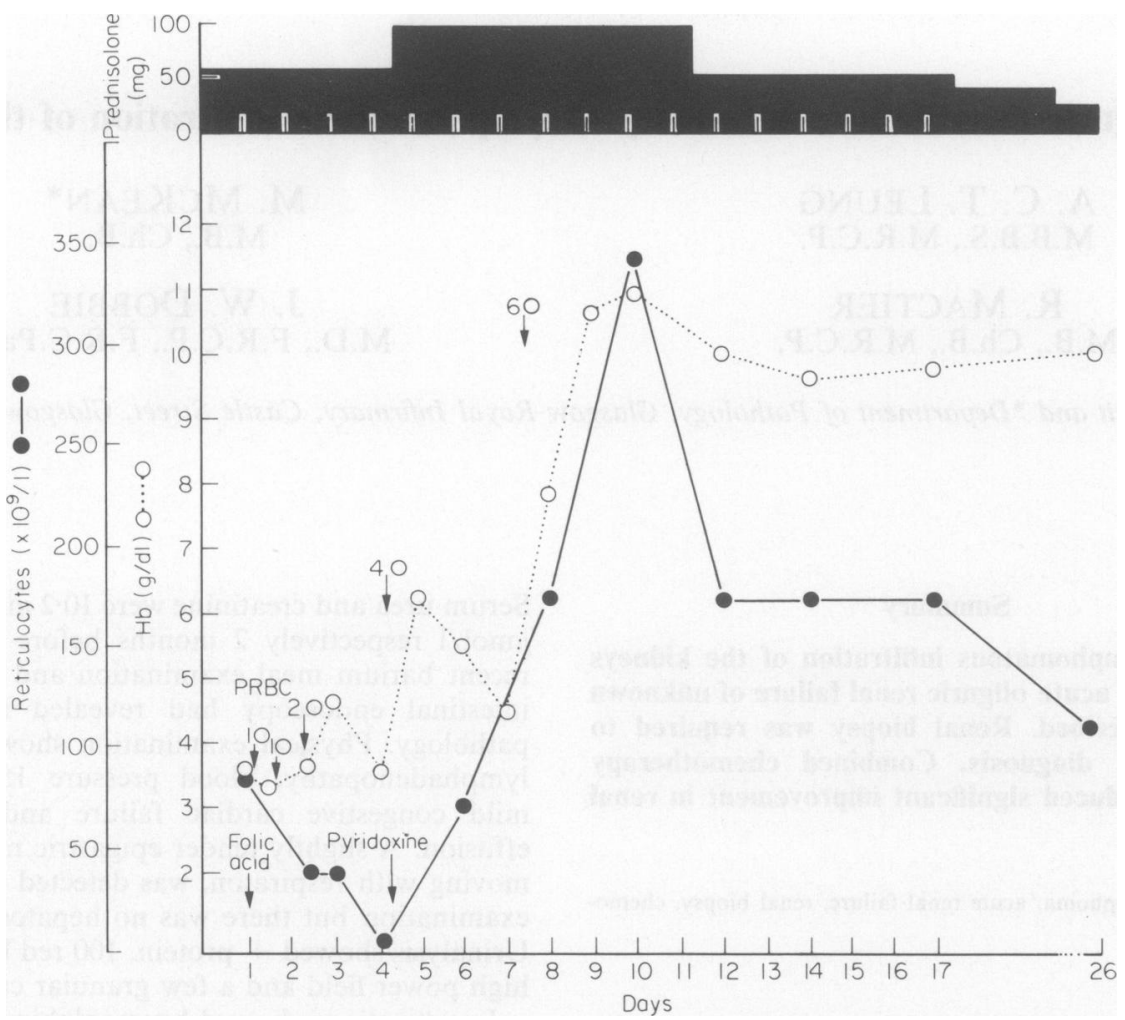

FIG. 1. Changes in haemoglobin concentration and reticulocyte count related to changes in therapy. Transfused packed red blood cells are shown (units $=$ PRBC).

Our case is noteworthy because previous and follow-up marrow aspirates showed no abnormality of the red cell precursors. It is likely that the marrow changes significantly increased the transfusion requirement in this haemolytic crisis. It also raises the question as to whether pyridoxine deficiency leading to sideroblastic change can occur in haemolytic crises.

\section{Acknowledgment}

We thank Dr K. R. Gough for permission to report this case.

\section{References}

Celada, A., Farquet, J.J. \& Muller, A.F. (1977) Refractory sideroblastic anaemia secondary to autoimmune haemolytic anaemia. Acta Haematologica (Basle), 58, 213.

Conley, C.L., Lippman, S.M., Ness, P.M., Petz, L.D., Branch, D.R. \& Gallagher, M.T. (1982) Autoimmune haemolytic anaemia with reticulocytopenia and erythroid marrow. New England Journal of Medicine, 306, 281.

MCFADZEAN, A.J.S. \& DAVIS, L.J. (1947) Iron staining erythrocytic inclusions with especial reference to acquired haemolytic anaemia. Glasgow Medical Journal, 28, 237.

(Accepted 23 June 1983) 